\title{
PLANTAS MEDICINAIS, MALÁRIA E POVOS INDÍGENAS: ESTUDOS ETNOBOTÂNICOS NO NORTE DA AMAZÔNIA
}

\author{
William Milliken ${ }^{1}$
}

RESUMO: Este trabalho apresenta os resultados de um levantamento das plantas medicinais utilizadas pelos índios Yanomami, e um levantamento das plantas utilizadas no tratamento da malária no Estado de Roraima. Em três comunidades Yanomami (Demini e Balawaú nas 'terras baixas' e Xitei nas 'terras altas'), aproximadamente 198 espécies de plantas medicinais foram coletadas, representando somente uma parte do conhecimento que os Yanomami possuem. Entre todos os grupos indígenas e comunidades visitadas, foram coletadas 99 espécies utilizadas no tratamento de malária.

Palavras-chave: Etnobotânica, Yanomami, plantas medicinais, plantas anti-maláricas.

ABSTRACT: This paper presents the overall results of a survey of medicinal plants used by the Yanomami Indians, and a survey of the plants used in the treatment of malaria in Roraima State. In three Yanomami comunities (at Demini and Balawaú in the lowlands and Xitei in the highlands), aproximately 198 species of medicinal plants were collected, representing only a part of the total Yanomami knowledge of this subject. Between all of the indigenous groups and non-indigenous communities visited, aproximately 99 species of plants were collected which are used in the treatment of malaria.

Key words: Ethnobotany, Yanomami, medicinal plants, antimalarial plants.

1 Pesquisador do Royal Botanic Gardens, Kew, Richmond, Surrey, Tw9 3AE, UK.

Bol. do Mus. Integrado de Roraima, Boa Vista, 4 (único) : 23-30, 1998 


\section{INTRODUÇÃO}

Malária

As tentativas de combater a malária ou paludismo estão ficando cada vez mais enfraquecidas, à medida que grupos resistentes ao parasita vão se desenvolvendo, tomando medicamentos com base em quinina, e outras drogas antimaláricas, ineficazes. De acordo com a Organização Mundial da Saúde (WHO), 270 milhões de pessoas são portadoras de paludismo e 2,1 bilhões (metade da população mundial) estão sujeitas à infecção (WHO, 1992; 1994). Somente no continente africano, calcula-se que um milhão de crianças morrem infectadas pela malária a cada ano, e esta cifra pode aumentar drasticamente no futuro se não for encontrada uma solução. Assim, a busca por novos agentes anti-maláricos é prioridade máxima (PHILLIPSON \& WRIGHT, 1991).

Plantas anti-maláricas

A maioria dos medicamentos com ação terapêtica contra a malária em uso hoje em dia é derivado sintético da quinina, uma substância natural originariamente extraída da casca de árvores de Cinchona spp. encontradas na América do Sul. Todavia, centenas de outras plantas são usadas por populações indígenas em todo o mundo para combater o paludismo, mas poucas foram submetidas a estudos farmacológicos aprofundados (SCHULTES \& RAFFAUF, 1994). Uma destas plantas (Artemisia annua - Asteraceae), usada na medicina tradicional na China, é altamente eficaz no combate aos recentes grupos de malária resistentes à cloroquina (KLAYMAN, 1985), e produtos farmacêuticos derivados dessa planta já estão sendo usados para combater a malária na Amazônia.

Os Yanomami

Os índios Yanomami, que habitam o norte da bacia amazônica (Brasil e Venezuela) e que representam o maior grupo indígena sobrevivente na Amazônia brasileira, estão sofrendo atualmente uma epidemia de malária com consequências graves. O paludismo é uma doença contra a qual este povo não tem nenhuma

Bol. do Mus. Integrado de Roraima, Boa Vista, 4 (único): 23-30, 1998 
resistência. A invasão de garimpeiros no final dos anos 80 , muitos dos quais oriundos de regiões onde o paludismo é endêmico, foi responsável pela disseminação da doença nas aldeias indígenas. Apesar de muitos dos garimpeiros já terem deixado a região, as doenças continuam ameaçando a tribo dos Yanomami .

Em alguns lugares o combate à epidemia através de medicamentos modernos não tem obtido muito sucesso. As aldeias dos Yanomami são dispersas, tornando impossível uma distribuição regular, e há uma distinta falta de recursos e pessoal disponíveis. Além disso, grupos de malária resistentes às drogas surgiram na região.

Existe uma alternativa nas plantas medicinais da região, que poderiam ser cultivadas ou coletadas na mata em todas partes do território indígena, constituindo uma fonte terapéutica permanente, e agindo como suplemento ou apoio aos programas de saúde.

Grande parte da medicina tradicional dos Yanomami é baseada no xamanismo (pajelança). No passado, no entanto, eles também utilizavam um número significativo de plantas medicinais. Em certas comunidades, a introdução de drogas modernas pelos missionários, agências governamentais e equipes de saúde, suplantou a maior parte da fitoterapia tradicional deste povo. Muito do conhecimento de utilização destas plantas está armazenado na memória de uns poucos curandeiros de idade avançada e, portanto, em risco de perda iminente (MILLIKEN \& ALBERT, 1996). A dependência total de medicamentos provenientes de fora apresenta um grande perigo para este povo, e o apoio à fitoterapia tradicional pode ser um importante fator preventivo.

Os objetivos básicos desta pesquisa foram os seguintes:

- Identificar plantas anti-maláricas regionais com maior potencial para uso por populações do interior; quais faltam, ou esporadicamente ou continuamente; disponibilidade de medicamentos eficientes ou apoio de saúde, quais precisam de uma alternativa viável.

- Realizar um levantamento geral do conhecimento de plantas medicinais dos Yanomami com propósito eventual de incentivar

Bol. do Mus. Integrado de Roraima, Boa Vista, 4(único): 23-30, 1998 
os médicos e enfermeiros, trabalhando entre eles, a considerar as plantas como uma alternativa ao uso contínuo de produtos farmacêuticos, e assim diminur a dependência que está sendo criada entre os Yanomami.

\section{MATERIAL E MÉTODOS}

Entre 1993 e 1995, o levantamento de plantas medicinais dos Yanomani foi realizado nas malocas de Watoriki (Demini), Watatasi e Kuai u (Xitei), e na Balawaú, através de entrevistas e coleta de material botânico para identificação das espécies utilizadas.

As entrevistas foram conduzidas usando as técnicas básicas que são universalmente empregadas na etnobotânica (incluindo verificação com pelo menos dois informantes, etc.), com tradução da língua Yanomami para Português feito pelo Antropólogo Bruce Albert (ORSTOM, França).

Amostras botânicas foram preservadas em álcool 70\% no campo (método Schweinfurth), e subsequentemente secas no Herbário do Museu Integrado de Roraima.

Entre 1994 e 1995 um levantamento das plantas usadas no tratamento de malária foi realizado entre grupos dos índios Macuxi, Wapixana, Wai-wai, Maiongong, Taurepang e Ingaricó, usando as mesmas técnicas descritas acima. Material de cada espécie (a parte da planta usada no preparo do remédio) também foi coletado, seco ao sol, para eventual análise das propriedades anti-maláricas.

\section{RESULTADOS E DISCUSSÃO}

Plantas medicinais dos Yanomami

Um total de 113 espécies de plantas e fungos foram coletadas em Demini. Estas espécies pertencem a 50 famílias botânicas. As famílias mais importantes em termos de espécies utilizadas foram: Leguminosae (sens. lat.) (09 espécies), Piperaceae (08 spp.), Araceae (06 spp.), Moraceae (06 spp.) e Rubiaceae (06 spp.).

As plantas são empregadas para uma variedade de doenças, das quais as mais importantes foram: febres, diarréia, dor de barriga, inflamação de olho (conjuntivite), dor de dente e

Bol. do Mus. Integrado de Roraima, Boa Vista, 4(único): 23-30, 1998 
gripe. Geralmente as plantas são usadas sozinhas, e várias espécies servem para mais de uma doença. A maioria são administradas em forma de infusão, banho ou decocção, com mais de 50\% sendo aplicada externamente. As partes da planta mais utilizadas são as folhas e/ou a entrecasca.

Certas espécies de plantas medicinais utilizadas pelos Yanomami são bem conhecidas na região norte da Amazônia, e uma proporção significativa das informações fornecidas pelos Yanomami sobre as propriedades das plantas são corroboradas pela literatura já existente sobre fitoterapia.

Tabela 1. Algumas plantas medicinais utilizadas pelos Yanomami *

\begin{tabular}{|c|c|c|c|}
\hline Família & Espécie & Nome vulgar & Uso \\
\hline Rubiaceae & $\begin{array}{l}\text { Uncaria guianensis (Aubl.) } \\
\text { Gmel. }\end{array}$ & $\begin{array}{l}\text { Cipó unha de } \\
\text { gato }\end{array}$ & Diarréia \\
\hline Leguminosae & Bauhinia guianensis Aubl. & $\begin{array}{l}\text { Cipó escada de } \\
\text { jabuti }\end{array}$ & Diarréia \\
\hline Apocynaceae & Aspidosperma nitidum Benth. & Carapanaúba & Malária \\
\hline Urticaceae & Urera baccifera (L.) Gaud. & Urtiga & Dor muscular \\
\hline Piperaceae & Piper arborea Aubl. & $?$ & Febre \\
\hline Rutaceae & $\begin{array}{l}\text { Zanthoxylum pentandrum } \\
\text { (Aubl.) R. Howard }\end{array}$ & ? & Dor de dente \\
\hline Piperaceae & $\begin{array}{l}\text { Peperomia rotundifolia (L.) } \\
\text { Kunth }\end{array}$ & ? & Tosse \\
\hline Anacardiaceae & $\begin{array}{l}\text { Anacardium giganteum Hancock } \\
\text { ex Engl. }\end{array}$ & Cajuí & Diarréia \\
\hline Leguminosae & Inga acuminata Benth. & Ingá & Sapinho \\
\hline Burseraceae & Protium fimbriatum Swart & Breu & Congetão nasal \\
\hline Bignoniaceae & $\begin{array}{l}\text { Jacaranda copaia (Aubl.) } \\
\text { D.Don }\end{array}$ & Caroba & $\begin{array}{l}\text { Picada } \\
\text { cobra }\end{array}$ \\
\hline Clusiaceae & Vismia angusta Miq. & Lacre & Pano branco \\
\hline $\begin{array}{l}\text { *Espécies } \\
\text { lugares }\end{array}$ & m propriedades medicina & já & as em out \\
\hline
\end{tabular}

Em Xitei e Balawaú foram coletadas aproximadamente 109 espécies de plantas medicinais, das quais somente 33 já tinham sido coletadas em Demini. Essa diferença é atribuível, pelo menos em parte, à diferença em altitude entre Demini (nas 'terras baixas') e Xitei (nas 'terras altas'), que se mostra

Bol. Mus. Int. de Roraima, Boa Vista, 4 (único): 23-30, 1998 
na composição florística da floresta (MILLIKEN \& ALBERT, 1997).

Plantas anti-maláricas de Roraima

Entre os grupos indígenas visitados no levantamento de plantas usadas no tratamento de malária (Yanomami, Maiongong, Macuxi, Wapixana, Ingaricó, Taurepang e Wai-wai) e também utilizadas pela população não indígena da região (incluindo fazendeiros, garimpeiros, etc.), um total de 99 espécies foram coletadas, incluíndo plantas tanto da floresta como do lavrado (MILLIKEN, 1997). Dessas espécies, poucas já foram pesquisadas quanto a propriedade anti-malárica, enquanto várias delas são comumente empregadas para os mesmos fins em outros Estados do Brasil ou em outros países. O nível de corroboração entre estes dados e as informações disponíveis na literatura existente é bastante alto.

o nível de conhecimento de plantas para tratamento de malária varia muito de uma comunidade para outra, denpendendo do nível da exposição para a comunidade que já tem a doença. Existe uma forte ligação entre a atribuição de propriedades anti-maláricas e a existência de um sabor amargo numa planta.

Tabela 2. Algumas espécies de plantas usadas no tratamento de malária em Roraima.

\begin{tabular}{lll}
\hline Espécies & Família & Nome vulgar \\
\hline \hline Aspidosperma nitidum Benth & Apocynaceae & Carapanaúba \\
Bidens bipinnata L. & Asteraceae & Picão \\
Carica papaya L. & Caricaceae & Mamão \\
Cissampelos ovalifolia DC. & Menispermaceae & Orelha de onça \\
Geissospermum sericeum (Sagot.) & Apocynaceae & Quina-quina \\
Benth \& Hook & & \\
Gossypum barbadense L. & Malvaceae & Algodão roxo \\
Himatanthus articulatus (Vahl) & Apocynaceae & Sucuba \\
Woods & & \\
Lippia schomburgkiana Schum. & Verbenaceae & Salva do campo \\
Momordica charantia L. & Cucurbitaceae & Melão São Caetano \\
Palicourea rigida Kunth & Rubiaceae & Douradão \\
Scoparia dulcis L. & Scrophulariaceae & Vassourinha \\
Senna occidentalis (L.) Link & Leguminosae & Mangerioba \\
Stachytarpheta cayennensis & Verbenaceae & Gefran \\
(Rich.) Vahl & & \\
Vernonia condensata Baker & Asteraceae & Boldo do Chile \\
\hline
\end{tabular}


O fato de ser usado no tratamento de malária não quer dizer necessariamente que uma espécie tem propriedades antimaláricas. Em muitos casos é provável que a planta seja usada para aliviar os sintomas da doença, em geral, inflamação do fígado e baço, febre etc. Espera-se que os testes de laboratório venham a ajudar a classificar esse problema, e mostrar as espécies que agem contra o parasita no corpo. Estes resultados, que ainda estão muito incompletos, serão divulgados numa publicação futura.

\section{AGRADECIMENTOS}

Essa pesquisa, feita em parte com Dr. Bruce Albert (ORSTOM, Paris), representa um projeto de colaboração entre a Universidade de Brasilia (Prof. Alcida Ramos como responsável), o Royal Botanic Gardens Kew (UK) e o Museu Integrado de Roraima. Recebeu ajuda inestimável das comunidades participantes, do CCPY, FNS (DSY), CIR, FUNAI, INPA, IBAMA, CNPq, APIR, e de vários profissionais, numerosos demais para mencionar aqui.

\section{BIBLIOGRAFIA}

KLAYMAN, D.L. (1985). Quinghaosu (Artemisinin): an antimalarial drug from China. Science 228: 1049-1055.

MILLIKEN, W. (1997). Traditional anti-malarial medicine in Roraima, Brazil. Economic Botany 51 (3): 212-237.

MILLIKEN, W. \& ALBERT, B. (1996). The use of medicinal plants by the Yanomami Indians of Brazil. Economic Botany 51 (1): $10-25$.

MILLIKEN, W. \& ALBERT, B. (1997). The use of medicinal plants by the Yanomami Indians of Brazil II. Economic Botany 51 (3) : 264-278.

PHILLIPSON, J.D. \& WRIGHT, C.W. (1991). Can ethnopharmacology contribute to the development of antimalarial agents? Journal of Ethnopharmacology 32: 155-165.

SCHULtes, R.E. \& Raffauf, R.F. (1994). De plantis toxicariis e mundo novo tropicale commentationes XXXIX. Febrifuges of northwest Amazonia. Harvard Papers in Botany 5: 52-68.

WHO (1992). World malaria situation 1990. World Health Bol. do Mus. Integrado de Roraima, Boa Vista, 4 (único): 23-30, 1998

Bol. do Mus. Integrado de Roraima, Boa Vista, 4 (único): 23-30, 1998 
Statistics Quarterly 45: 257-266.

WHO (1994). World malaria situation in 1992. Weekly Epidemiological Record 42: 309-314.

Recebido em: 01.03.97

Aceito em: 12.09 .97

Bol. do Mus. Intearado de Roraima, Boa Vista, 4(único): 23-30, 1998 